\title{
Variations
}

Variations

Revue internationale de théorie critique

$8 \mid 2006$

Subjectivités libres et critique de la répression

\section{Theodor Wiesengrund Adorno, La Métaphysique. Concept et problèmes}

Alexander Neumann

\section{(2) OpenEdition}

1 Journals

Édition électronique

URL : http://journals.openedition.org/variations/516

DOI : 10.4000/variations.516

ISSN : 1968-3960

Éditeur

Les amis de Variations

Édition imprimée

Date de publication : 1 novembre 2006

Pagination : 108-110

Référence électronique

Alexander Neumann, "THEOdor WIESENgRUNd AdoRno, LA MÉTAPHYSIQUe. CONCEPT ET PROBLÈMES 》, Variations [En ligne], 8 | 2006, mis en ligne le 27 décembre 2012, consulté le 22 septembre 2020. URL :

http://journals.openedition.org/variations/516 ; DOI : https://doi.org/10.4000/variations.516

Ce document a été généré automatiquement le 22 septembre 2020.

Les ami•e•s de Variations 


\title{
Theodor Wiesengrund Adorno, $L a$ Métaphysique. Concept et problèmes
}

\author{
Alexander Neumann
}

\section{RÉFÉRENCE}

Theodor W. Adorno, La Métaphysique. Concept et problèmes, Traduction et présentation par Christophe David, Payot, collection Critique de la Politique, Paris, 2006

1 La légende veut qu'Adorno, qui compte parmi les illustres fondateurs de la Théorie critique, ait fermé la porte de la petite salle de son séminaire de recherche, dès que les onze places disponibles aient été prises, dans une sorte de jeu de chaises musicales universitaire. S'il est connu que la théorie de la démocratie et sa pratique convergent rarement, la véracité de l'anecdote reste cependant à établir. Peu importe au fond, car les lecteurs de la Métaphysique jouissent tous du délicieux privilège d'assister en direct aux conférences adorniennes: l'ouvrage est la retranscription fidèle des cours magistraux que le philosophe a présentés devant les étudiants de l'Université de Francfort à l'été 1965. Les dix-huit chapitres du livre se font ainsi l'écho d'autant de séances, témoignant des rappels, précisions et reprises du professeur, qui affiche ses talents pédagogiques et une rare humanité. Contrairement à l'impression très académique que provoque le titre, La Métaphysique reconstitue une parole vivante, ouverte et souvent ironique. Parmi les phrases introductives, on trouve ainsi ce constat dépité : «De nos jours, la métaphysique passe pour un gros mot, à peu près dans le monde entier mis à part l'Allemagne, et qu'on associe à une sorte de spéculation vaniteuse, à un simple jeu de l'esprit ou à Dieu sait quel autre péché intellectuel."

2 L'auteur rappelle que chez Nietzsche, la métaphysique est identifiée aux habitants des arrière-bois de l'Ouest américain, dans le double sens de la province perdue et d'une pensée supposant qu'il existe un monde derrière le monde que nous éprouvons. La question est de savoir ce qui se trouve au-delà du monde sensible. Voilà comment Adorno introduit le concept philosophique de transcendance, qui se distingue, par 
exemple, de l'obscurantisme astrologique. Élisabeth Tessier sera ravie de l'apprendre. Rapidement, l'exposé s'oriente vers le rapport fondamental entre, d'un côté, le monde empiriquement saisissable et, de l'autre, les concepts qui nous permettent de le nommer. Adorno touche le noyau du problème, déjà présent chez Aristote : les concepts sont-ils des simples signifiants d'une réalité factuelle, ou possèdent-ils leur propre existence substantielle? En quelques mots, le philosophe francfortois saisit la querelle du nominalisme qui pointe ici, en passant par les philosophes de l'Antiquité grecque et par l'idéalisme allemand. Dans un même souffle, il renverse le positivisme sociologique et sa manie des définitions formelles, d'Auguste Comte au 11 mai 1965, date à laquelle il donne le cours dont il est question. En somme, la métaphysique décrit toujours des doctrines qui supposent que les concepts constituent une sorte d'objectivité propre, alors que la Théorie critique interroge ce lien entre sujet et objet. Selon Adorno, l'expérience singulière échappe toujours au concept, à des degrés divers, alors que les multiples faits qui constituent le monde menacent, inversement, de nous étouffer par leur réalisme s'ils ne sont pas mis à distance par des conceptualisations critiques. Cette argumentation originale et forte guide tout le cycle de cours, rappelant sans cesse la thèse de la dialectique négative ${ }^{1}$, élaborée quelques années auparavant, notamment en opposition à la dialectique historique de Hegel. Chez l'auteur de la Phénoménologie de l'Esprit, le mouvement dialectique du monde et des concepts donne forme à la société et à l'État, faisant converger sujet et objet grâce au travail de l'esprit. L'esprit du monde s'affirme ici progressivement et positivement à travers l'histoire, aboutissant à une identité totalisante de la société. Adorno constate que pareille fusion est impossible, à moins de taire tout ce qui échappe au langage pur des concepts, c'est-à-dire en refoulant les particularités et éléments contradictoires de la vie. C'est un dilemme connu de l'écriture, où chaque phrase écrite omet une multitude de détails, d'aspects et de nuances. Ces aspects subordonnés ou écartés constituent une négativité face à l'ordre positif du monde et du fait accompli, négativité qui rend obsolète l'idée d'une totalisation historique définitive, d'une fin de l'histoire. Vers la fin de l'ouvrage, après une série de détours éclairants, Adorno aborde trois subterfuges théoriques, qui prétendent échapper à cette tension permanente du particulier et de la totalisation, de la positivité et de ses négations. D'abord, il rappelle que le rejet de la métaphysique revient à reproduire ses propres travers, car on rejette alors un concept au lieu de saisir le problème, tout en restant précisément enfermé dans le cadre théorique de la métaphysique. Sur un autre plan, même Marx bute sur le caractère revêche de la négativité, lorsqu'il s'imagine que la négation de la négation puisse produire une humanité réconciliée avec elle-même, autrement dit une totalisation sans reste.

3 Enfin, la critique adornienne se moque des auteurs contemporains qui dénoncent avec verve la réification des relations sociales et du langage. Car les éléments rigidement objectifs prouvent que nul processus d'identification, aussi dialectique soit-il, n'arrive à dissoudre toutes les objectivations, à moins de dériver vers une pensée subjectiviste de la volonté pure. L'objectivité rigide des catégories de pensée de la philosophie métaphysique exprime non seulement une "société rigidifiée ", mais rappelle aussi la "primauté de l'objet» devant la pensée, comme le souligne l'auteur dans le dernier chapitre.

4 L'exposé au sujet de la métaphysique concentre ainsi un ensemble de discours fondateurs de la Théorie critique, qui se construit contre l'idéalisme spéculatif, contre le positivisme froid et contre l'existentialisme pathétique. Ce condensé critique se lit d'une manière étonnamment facile, grâce au style accessible que l'auteur a choisi en 
face des jeunes intellectuels qu'il trouve en face de lui. Ce public est à la recherche d'arguments qui échappent à l'idéologie bureaucratique du marxisme soviétique, tout comme à l'emprise réactionnaire du jargon heideggerien, hier comme aujourd'hui. Nous savons que la pensée d'Adorno, loin de se limiter à des exercices philosophiques, a puissamment stimulé la révolte anti-autoritaire des étudiants allemands, qui éclate d'ailleurs à peine deux ans après le cours sur la métaphysique. Cela montre que l'émancipation peut jaillir de la déconnexion de la théorie et de la pratique, alors que leur fusion l'engloutit à coup sûr.

5 Reste à souhaiter que le caractère accessible de l'ouvrage, introduit par Christophe David, ébranle encore un peu plus les résistances françaises à la réception de la Théorie critique, résistances qui furent longtemps le fait d'une Sainte alliance entre staliniens et conservateurs. Parmi les ouvrages tirés des conférences d'Adorno, établis au sein d'une collection qui regroupe les publications posthumes (Suhrkamp, Francfort), un autre cours donné en 1960 mériterait particulièrement d'être traduit : La Philosophie et la sociologie. La Théorie critique, sommée de choisir entre les disciplines académiques, a toujours pris le chemin du dépassement et de la transgression des catégories traditionnelles. Comme la Métaphysique adornienne le montre, il s'agit de formuler une mise en relation fondamentalement différente du concept et de l'expérience.

\section{NOTES}

1. Adorno, La Dialectique négative, Payot, 2003. 